\title{
Theoretical amplitudes and lifetimes of non-radial solar-like oscillations in red giants
}

\author{
M.-A. Dupret ${ }^{1,2}$, K. Belkacem ${ }^{2,1}$, R. Samadi ${ }^{1}$, J. Montalban ${ }^{2}$, O. Moreira ${ }^{2}$, A. Miglio ${ }^{2}$, M. Godart ${ }^{2}$, P. Ventura ${ }^{3}$, \\ H.-G. Ludwig ${ }^{\star, 4}$, A. Grigahcène ${ }^{5}$, M.-J. Goupil ${ }^{1}$, A. Noels ${ }^{2}$, and E. Caffau ${ }^{4}$ \\ 1 LESIA, Observatoire de Paris, CNRS UMR 8109, Université Paris Diderot, 5 place J. Janssen, 92195 Meudon, France \\ 2 Institut d'Astrophysique et de Géophysique de l'Université de Liège, Allée du 6 Août 17, 4000 Liège, Belgium \\ e-mail: ma.dupret@ulg.ac.be \\ INAF - Osservatorio Astronomico di Roma, MontePorzio Catone (RM), Italy \\ 4 GEPI, Observatoire de Paris, CNRS, Université Paris Diderot, 92195 Meudon Cedex, France \\ ${ }^{5}$ Centro de Astrofisica da Universidade do Porto, Rua das Estrelas, 4150-762 Porto, Portugal
}

Received 23 January 2009 / Accepted 3 April 2009

\begin{abstract}
Context. Solar-like oscillations have been observed in numerous red giants from ground and from space. An important question arises: could we expect to detect non-radial modes probing the internal structure of these stars?

Aims. We investigate under what physical circumstances non-radial modes could be observable in red giants; what would be their amplitudes, lifetimes and heights in the power spectrum (PS)?

Methods. Using a non-radial non-adiabatic pulsation code including a non-local time-dependent treatment of convection, we compute the theoretical lifetimes of radial and non-radial modes in several red giant models. Next, using a stochastic excitation model, we compute the amplitudes of these modes and their heights in the PS.

Results. Distinct cases appear. Case A corresponds to subgiants and stars at the bottom of the ascending giant branch. Our results show that the lifetimes of the modes are mainly proportional to the inertia $I$, which is modulated by the mode trapping. The predicted amplitudes are lower for non-radial modes. But the height of the peaks in the PS are of the same order for radial and non-radial modes as long as they can be resolved. The resulting frequency spectrum is complex. Case B corresponds to intermediate models in the red giant branch. In these models, the radiative damping becomes high enough to destroy the non-radial modes trapped in the core. Hence, only modes trapped in the envelope have significant heights in the PS and could be observed. The resulting frequency spectrum of detectable modes is regular for $\ell=0$ and 2 , but a little more complex for $\ell=1$ modes because of less efficient trapping. Case $\mathrm{C}$ corresponds to models of even higher luminosity. In these models the radiative damping of non-radial modes is even larger than in the previous case and only radial and non-radial modes completely trapped in the envelope could be observed. The frequency pattern is very regular for these stars. The comparison between the predictions for radial and non-radial modes is very different if we consider the heights in the PS instead of the amplitudes. This is important as the heights (not the amplitudes) are used as detection criterion.
\end{abstract}

Key words. stars: oscillations - stars: interiors - convection

\section{Introduction}

Oscillations of high radial order p-modes have been firmly detected in several red giants, from radial velocity data (Frandsen et al. 2002; Bedding \& Kjeldsen 2006) and photometric data from space (Barban et al. 2007; De Ridder et al. 2009). These oscillations are stochastically excited by turbulent motion in the outer layers of these stars. A particularly interesting case is $\epsilon$ Oph, in which De Ridder et al. (2006) detected equidistant frequencies from ground-based spectroscopic observations. Hekker et al. (2006) analysed the line-profile variations and advocate the presence of non-radial modes. For the same star, Barban et al. (2007) used data obtained by the MOST satellite. They detected at least 7 equidistant peaks and derived mode lifetimes of about 2.7 days. Kallinger et al. (2008a) reexamined the available data for $\epsilon$ Oph and claim to detect at least 21 independent modes with longer lifetimes between 10 and 20 days. Kallinger et al. (2008b)

^ CIFIST Marie Curie Excellence Team. claim to have detected non-radial modes with long lifetimes (more than 10 days) in the star HD 20884 observed by MOST. A very different result was found by Stello et al. (2006) who claim to have detected modes with very short lifetimes in $\xi$ Hydrae. We finally mention the recent detection by the CoRoT satellite of solar-like radial and non-radial oscillations in many red giants (De Ridder et al. 2009). Different kinds of power spectra are found in these data of unprecedented quality, some of them showing regular patterns while others are more complex. Modes with very long lifetimes (more than 50 days) are detected.

From a theoretical point of view, an important study was made by (Dziembowski et al. 2001, hereafter D01). They underline the mode trapping phenomenon present in these stars and the role of radiative damping for several models with different luminosities. They predict that non-radial modes not trapped in the envelope would have much smaller amplitudes than radial modes. Houdek \& Gough (2002) carried out theoretical computations of the amplitudes and lifetimes of radial modes in the 
star $\xi$ Hydrae. They found lifetimes of the order of 15-20 days with a bump going up to infinite value (unstable mode) around $110 \mu \mathrm{Hz}$. Finally, Christensen-Dalsgaard (2004) also discuss the mode trapping and inertia of non-radial adiabatic modes in red giants and subgiants and the possible consequences for the amplitudes.

In the present study, we compute theoretical lifetimes of radial and non-radial modes of several red giant models, using a fully non-radial non-adiabatic pulsation code including the nonlocal time-dependent treatment of convection by Grigahcène et al. (2005) (G05). We discuss how these lifetimes are affected by the mode inertia, the radiative damping in the core and the convective damping in the upper part of the convective envelope. Using a stochastic excitation model (Samadi \& Goupil 2001; Samadi et al. 2003b,a), we also compute the amplitudes of radial and non-radial modes and their theoretical heights in the power spectrum (PS). As we show below, predictions for the heights are very different from the amplitudes. Although non-radial modes in general have smaller amplitudes than radial modes, this is not always the case for the heights. As the heights in the PS are used as a criterion for the detection of frequencies, it is important to make this distinction.

In Sect. 2, we discuss the different energetic processes determining the driving and damping of the modes: the radiative damping in the core, the coherent interaction of convection with oscillations and the stochastic excitation by turbulent motion in the upper part of the convective envelope. In Sect. 3, we present the numerical tools, models and method used to solve the problem. In Sect. 4, we present our results for specific red giant models representative of the different cases that can occur.

\section{Energetic aspects of oscillations}

Different mechanisms lead to the driving and damping of oscillations. For observed modes, two possibilities exist. Firstly, they could be self-excited. In such case the amplitudes are expected to grow until a large amplitude limit cycle is reached. The observed amplitudes of the high radial order p-modes in red giants are of several $\mathrm{m} / \mathrm{s}$. This is too small for such an interpretation and we reject this possibility here. Secondly, the modes can be damped, but turbulent motions supply them with energy in a stochastic way, allowing them to reach observed amplitudes. We assume here that the high frequency modes of red giants are stochastic excited modes. The damping rate of the modes is given by an integral expression of this type:

$\eta=-\frac{\int_{V} \mathrm{~d} W}{2 \sigma I\left|\xi_{\mathrm{r}}(R)\right|^{2} M}$,

where we assumed the time-dependence $\exp (\mathrm{i} \sigma t-\eta t)(t$ is the time, $\sigma$ is the angular frequency and $\eta$ is the damping rate). $\int \mathrm{d} W$ is the work performed by the gas during one oscillation cycle. $\boldsymbol{\xi}$ is the displacement vector, $I$ is the dimensionless mode inertia:

$I=\int_{0}^{M}|\xi|^{2} \mathrm{~d} m /\left(M\left|\xi_{\mathrm{r}}(R)\right|^{2}\right)$,

$M$ is the total mass and $\xi_{\mathrm{r}}(R)$ corresponds to the radial displacement at the layer where the oscillations are measured (Rosseland optical depth $\tau_{\mathrm{R}}=0.1$ in our results). In radiative zones, the work is easily modeled. It just results from the heat given to the gas by radiation during each oscillation cycle. In convective zones, the problem is much more complex and different terms can lead to damping: variations of convective flux, turbulent pressure, viscosity, and dissipation of turbulent kinetic energy (G05). Stochastic excitation by turbulent motion also occurs, leading to the observed amplitudes. This is discussed in Sect. 2.3.

\subsection{Radiative damping}

In the g-mode cavity of red giants, the radial wave-number $k_{\mathrm{r}}=$ $\sqrt{\ell(\ell+1)} N /(\sigma r)$ becomes huge because of the high density contrast between the core and the envelope ( $N$ is the Brunt-Väisälä frequency). Large variations of the temperature gradient ensue, with loss of heat in the hot phase, leading to radiative damping. In the asymptotic limit, a simple expression for this damping can be obtained (Dziembowski 1977b; Van Hoolst et al. 1998; Godart et al. 2009):

$-\int_{r_{0}}^{r_{\mathrm{c}}} \frac{\mathrm{d} W}{\mathrm{~d} r} \mathrm{~d} r \simeq \frac{K[\ell(\ell+1)]^{3 / 2}}{2 \sigma^{3}} \int_{r_{0}}^{r_{\mathrm{c}}} \frac{\nabla_{\mathrm{ad}}-\nabla}{\nabla} \frac{\nabla_{\mathrm{ad}} N g L}{p r^{5}} \mathrm{~d} r$,

where $r_{0}$ is the radius of the convective core (if present), $r_{\mathrm{c}}$ is the upper radius of the g-mode cavity, $K$ is a normalization constant obtained by appropriate matching with the envelope solution, $\nabla_{\mathrm{ad}}$ and $\nabla$ are the adiabatic and real gradients, $g$ the gravity, $L$ the local luminosity and $p$ the pressure. In the central regions of red giants, $\mathrm{Ng} / \mathrm{r}^{5}$ is very high because of the high density contrast. But at the same time, $p$ increases rapidly as we enter in the dense pure He core. As a compromise between these two tendencies, the integrand of Eq. (3) reaches its largest values around the bottom of the H-burning shell. The main radiative damping thus occurs in this region for red giants.

On the other side, the contribution of the g-mode cavity to the denominator of Eq. (1) is simply:

$8 \pi \sigma \int_{r_{0}}^{r_{\mathrm{c}}}|\xi|^{2} r^{2} \rho \mathrm{d} r \simeq 4 \pi K \int_{r_{0}}^{r_{\mathrm{c}}} k_{\mathrm{r}} \mathrm{d} r$

which also can be significant because of the large $N\left(k_{\mathrm{r}} \propto N\right.$ in the core). Equation (3) scales like $\tau_{\mathrm{KH}}^{-1}\left(R / R_{\mathrm{c}}\right)^{7 / 2}\left(M / M_{\mathrm{c}}\right)^{1 / 2}$, where $\tau_{\mathrm{KH}}=G M^{2} /(L R)$ is the Kelvin-Helmholtz time, $R_{\mathrm{c}}$ is the radius of the He core, $R$ is the total radius, $M_{\mathrm{c}}$ is the mass of the He core and $M$ is the total mass. Equation (4) scales like $\left(M_{\mathrm{c}} / M\right)^{1 / 2}\left(R / R_{\mathrm{c}}\right)^{3 / 2}$. As the star climbs the red giant branch, $L$ and $R / R_{\mathrm{c}}$ increase so that the numerator increases more quickly than the denominator and the radiative damping of non-radial modes increases.

\subsection{Coherent interaction with convection}

A very important region for the driving and damping of the modes is the transition region where the thermal relaxation time is of the same order as the oscillation periods. For solar-like oscillations, this transition region corresponds to the upper part of the convective envelope. Moreover, in this region the time-scale of most energetic turbulent eddies is of the same order as the oscillation periods. Hence, the changing physical conditions due to the oscillations lead to periodic variations of the turbulent quantities (convective flux, Reynolds stress, ...), which contribute to the work (positive or negative) performed by the modes. This is what we call the coherent interaction between convection and oscillations. It plays a major role in the damping of high radial order p-mode oscillations of red giants.

This interaction is difficult to model and only a few theories have been proposed for linear oscillations. Two of them make use of a Mixing-Length formalism. The first by Gabriel (1996) and further developed by G05 follows the original ideas of 
Unno (1967), where a turbulent viscous term opposite to the buoyancy is introduced. This treatment implemented in our nonradial non-adiabatic pulsation code is used in this study. The second theory was developed by Gough (1977b) and follows the "kinetic of gas" picture of the MLT. Both theories can include a non-local treatment (Spiegel 1963; Gough 1977a; Balmforth 1992; Dupret et al. 2006b). A third formulation, no longer based on a mixing-length approach but on a Reynolds stress one was also proposed by Xiong et al. (1997). These theories encountered some successes, for example they obtain the red edge of the instability strip (Houdek 2000; Xiong \& Deng 2001; Dupret et al. 2004). The reproduction of the Solar mode lifetime is also possible but not easy (Balmforth 1992; Xiong et al. 2000; Dupret et al. 2006a).

\subsection{Stochastic excitation}

For stochastically excited modes, the local squared amplitude of velocity variation at the layer where it is measured (we adopt the optical depth $\tau_{\mathrm{R}}=0.1$ in our results) is given by (e.g., Baudin et al. 2005; Belkacem et al. 2006):

$V^{2}=\frac{P}{2 \eta M I}=\frac{\Pi}{2 \eta M I^{2}}$,

$P$ is the power stochastically supplied to the modes:

$P=\frac{1}{8 M I}\left(C_{\mathrm{R}}^{2}+C_{S}^{2}\right)$,

where $C_{\mathrm{R}}^{2}$ and $C_{\mathrm{S}}^{2}$ are the turbulent Reynolds stress and entropy contributions, respectively (see Samadi \& Goupil 2001; Belkacem et al. 2006, for details). Both play a significant role in red giants and are included in our study. $\eta$ and $I$ are the damping rate and inertia defined in Eqs. (1) and (2), respectively. To isolate the effect of inertia in Eq. (5), we introduce the product $\Pi=P I$ which is, according to Eq. (6), independent of inertia.

We also introduce the maximum height of the mode profile in the PS, which is an observable and will permit us to draw conclusions about the mode detectability (see Sect. 4 for details). To this end, one has to distinguish between two cases, namely resolved and unresolved modes. The resolved modes present a Lorentzian profile in the PS and their heights are given by (see e.g. Chaplin et al. 2005; Belkacem et al. 2006):

$H=\frac{V^{2}(R)}{\eta}=\frac{\Pi}{2 \eta^{2} M I^{2}}=\frac{\Pi \tau^{2}}{2 M I^{2}}$

where $\tau=1 / \eta$ is the mode lifetime.

When $\tau \gtrsim T_{\mathrm{obs}} / 2$ ( $T_{\mathrm{obs}}$ being the duration of observations), the modes are not resolved. In the limit $\tau \rightarrow \infty$, the heights in the PS tend to behave like (see e.g. Berthomieu et al. 2001; Lochard et al. 2005):

$H_{\infty}=\frac{T_{\mathrm{obs}}}{2} V^{2}(R)=\frac{\Pi T_{\mathrm{obs}} \tau}{4 M I^{2}}$.

In the theoretical predictions of the heights presented in this paper, we choose the very favorable case of the CoRoT long runs with $T_{\text {obs }}=150$ days. We use Eq. (7) when $\tau \leq T_{\text {obs }} / 2=75$ days and Eq. (8) when $\tau>T_{\text {obs }} / 2=75$ days, so that $H$ is a continuous function of $\tau$.

Finally, the observed velocity amplitudes are obtained by integration of the projected local velocity over the visible stellar disk (Dziembowski 1977a). This introduces a visibility factor depending on the inclination angle of the star (through the factor $\left.P_{\ell}^{m}(\cos i)\right)$. We do not include this factor in our theoretical study (the inclination angle is unknown).
Table 1. Global parameters of our models.

\begin{tabular}{ccccccc}
\hline \hline & $M / M_{\odot}$ & $\begin{array}{c}T_{\text {eff }} \\
(\mathrm{K})\end{array}$ & $\log \left(L / L_{\odot}\right)$ & $\log g$ & $R / R_{\odot}$ & $\begin{array}{c}v_{\text {cut }} \\
\left(\mu_{\mathrm{Hz}}\right)\end{array}$ \\
\hline $\mathrm{A}$ & 2 & 5264 & 1.32 & 3.26 & 5.50 & 315 \\
$\mathrm{~B}$ & 2 & 4892 & 1.80 & 2.65 & 11.0 & 82.1 \\
$\mathrm{C}$ & 2 & 4665 & 2.10 & 2.27 & 17.2 & 34.6 \\
$\mathrm{D}$ & 3 & 5091 & 2.00 & 2.70 & 12.8 & 88.6 \\
$\mathrm{E}$ & 3 & 5222 & 2.00 & 2.74 & 12.2 & 96.3 \\
\hline
\end{tabular}

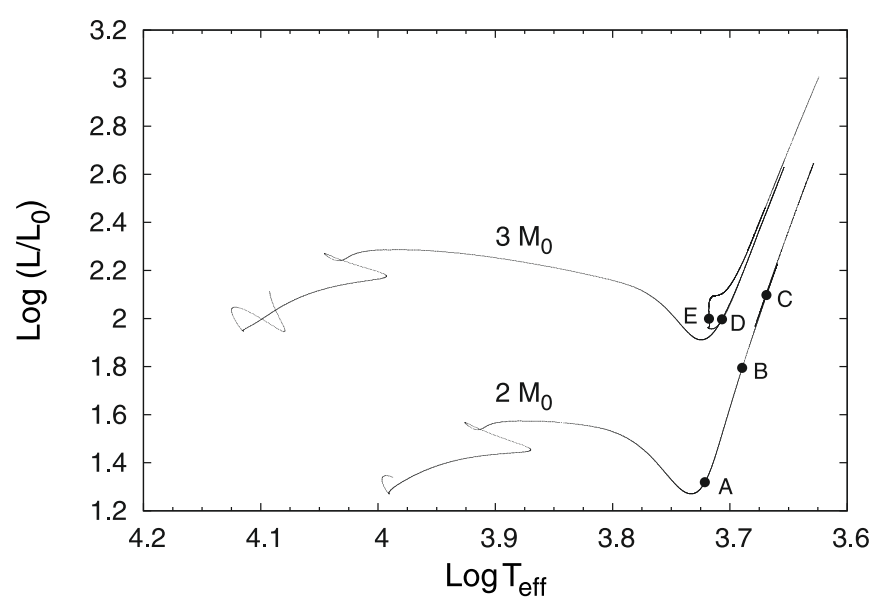

Fig. 1. HR diagram with the location of our five models.

\section{Numerical tools, models and method}

\subsection{Structure models}

The equilibrium models analyzed in this study were computed with the version updated for asteroseismology of the code ATON (Mazzitelli 1979). A detailed description of the numerical techniques and implemented physics is given in Ventura et al. (2008) and references therein. For the models in this paper we adopted the classical mixing-length theory of convection (Böhm-Vitense 1958), with a value of the mixing-length parameter $\alpha_{\mathrm{MLT}}=1.8$, and we assume core overshooting that was treated as a diffusive mixing process. For the parameter describing the extension of that extra mixing we took $\alpha_{\mathrm{OV}}=0.015$ (see Ventura et al. 1998, for details), and for the chemical composition $X=0.72$ and $Z=0.012$. The radiative opacities are those from OPAL (Iglesias \& Rogers 1996) for the metal mixture of Grevesse \& Noels (1993) completed with Alexander \& Ferguson (1994) at low temperatures, and the conductive ones come from Potekhin et al. (1999). Thermodynamic quantities are derived from OPAL (Rogers \& Nayfonov 2002), Saumon et al. (1995) for the pressure ionization regime and Stolzmann \& Bloecker (1996) treatment is used for He/C/O mixtures. The nuclear cross-sections are from the NACRE compilation (Angulo et al. 1999), and the surface boundary conditions are provided by a grey atmosphere following the Henyey et al. (1965) treatment. Turbulent pressure is not included in our structure models.

We focus our study on five well chosen models representative of the different situations that can occur. Some of their global characteristics are given in Table 1. $v_{\text {cut }}=(g / 2) \sqrt{\Gamma_{1} \rho / p}$ is the cut-off frequency of an isothermal plane-parallel atmosphere (estimated here at the photosphere). Evolutionary tracks with the location of these models in the HR diagram are given in Fig. 1. 


\subsection{Non-radial non-adiabatic oscillations}

We use the non-adiabatic pulsation code MAD (Dupret 2002) to compute the theoretical lifetimes and inertia of the modes. We use the TDC treatment of G05 for the modelling of the convective flux and turbulent pressure variations entering in the nonadiabatic equations. Different ingredients must be specified. We keep a local treatment for the convective flux. The variations of the turbulent pressure and entropy gradient are treated in a nonlocal way, with values of the non-local parameters $b=3$ and $c=3.5$ according to the definition of Balmforth (1992). Our value of $b$ is derived from a fit of the turbulent pressure in the subadiabatic atmospheric layers of a solar hydrodynamic simulation (Dupret et al. 2006b). We recall that our structure models are built without turbulent pressure. We compute it a posteriori in order to be able to determine the turbulent pressure perturbation. This implies that the effect of turbulent pressure on the stratification is not taken into account in our models. However, the impact of this inconsistency on the theoretical lifetimes is expected to be negligible compared to others aspects. The main source of uncertainties in any TDC treatment comes from the perturbation of the closure equations. In our TDC treatment, this uncertainty appears in the form of a free parameter $\beta$, as introduced by G05. We take by default the value $\beta=-2 i$. Adopting this value for the Sun leads to predictions of the mode lifetimes in reasonable agreement with observations. It also gives in all our red giant models stable high radial order p-modes, in agreement with our working hypothesis of stochastic excitation. However, we discuss in Sect. 4.6 the impact of changing this value.

Some changes were required in the MAD code to address specific numerical difficulties associated with red giants. First, we changed the formalism to a Eulerian one in the core. In the g-mode cavity of red giants where $\sigma \ll L_{\ell}$, we have $\left|p^{\prime} / p\right| \ll$ $|\delta p / p|$ and thus $\delta p / p \simeq(\mathrm{d} \ln p / \mathrm{d} r) \xi_{\mathrm{r}}$. Hence, $\delta p$ and $\xi_{\mathrm{r}}$ are not independent functions and they do not allow us to capture the oscillatory behaviour of the eigenfunctions in the cavity. Therefore, a Lagrangian formalism lacks numerical precision in the dense region of evolved stars, which justifies our switch to a Eulerian formalism.

Another significant numerical difficulty comes from the very large number of nodes of the eigenfunctions in the g-mode cavity. Van Hoolst et al. (1998) and D01 used a mixed treatment to address this difficulty: numerical solutions of the fully nonadiabatic equations in the envelope are matched with asymptotic solutions in the g-mode cavity. Our way to proceed is slightly different. Firstly we solve the fully non-adiabatic equations from the center to the surface, which gives a first estimation of the lifetimes. Second, we separate the integrals in Eqs. (1) and (2) in two parts: region 1 where $\sigma \ll L_{\ell}, N$ and region 2 (the remaining). In region 1 we use the results obtained in the asymptotic quasi-adiabatic limit (Eqs. (3) and (4)), and in region 2 we use the fully non-adiabatic eigenfunctions obtained numerically in the first step. We then compare the results obtained by the two approaches: in many cases they are very close, showing that our fully numerical solution of the problem is precise enough. In other cases corresponding to high luminosity red giants, the number of nodes becomes huge ( $\gtrsim 1000)$, which implies loss of numerical precision. In such cases we are more confident in using results with the asymptotic treatment in the g-mode cavity and the fully non-adiabatic solution in the rest of the star.

A last important numerical issue is associated with the mode trapping phenomenon, which is discussed in detail in Sect. 4.1. Algorithms solving the non-adiabatic problem converge towards the closest eigenvalue in the complex plane (the real part corresponds to the angular frequency $\sigma$ and the imaginary part to the damping rate $\eta$ ). Some non-radial modes trapped in the envelope have much lower inertia than the others. Hence, their damping rates are much higher than for other non-radial modes, higher than the frequency separation between consecutive nonradial modes: $\eta>\sigma_{n-1}-\sigma_{n}$. Hence, with a real initial guess of the eigenvalues (e.g. the adiabatic frequencies), it is impossible to converge towards the modes strongly trapped in the envelope. The only way is to adopt a complex initial value; for example, interpolating the complex non-adiabatic eigenvalues of radial modes gives adequate initial values, allowing the solution to converge towards the non-radial modes strongly trapped in the envelope.

\subsection{Ingredients for the stochastic excitation models}

The power stochastically supplied to the modes, $P$ in Eqs. (5) and (6), is computed as described in Belkacem et al. (2008). The typical convective length-scales as well as the kinetic energy spectrum are poorly known for red giant stars. Given that the predictions of the stochastic excitation models strongly depend on them, we infer both the kinetic energy spectrum and the injection length-scale from a representative 3D numerical simulation. To this end, we computed a 3D radiation-hydrodynamical model atmosphere with the code CO $^{5}$ BOLD (Freytag et al. 2002; Wedemeyer et al. 2004). The simulation has a gravity $\log g=2.5$ and an effective temperature of $T_{\text {eff }}=4960 \mathrm{~K}$. The model has a spatial mesh with $160 \times 160 \times 200$ grid points, and a physical extent of the computational box of $573 \times 573 \times 243 \mathrm{Mm}^{3}$. Using this $3 \mathrm{D}$ numerical simulation, we determine the injection length-scale in the layer where the driving is the largest (see Samadi et al. 2003b, for details). This estimation of the injection scale gives values larger than the mixing-length by a factor of 510 depending on the model. Using the mixing-length as the injection scale would give theoretical amplitudes much lower than typical observations. We assume here that the injection scale is constant in the driving region. In the case of the Sun, such approximation underestimates by about $25 \%$ the theoretical amplitudes (Samadi et al. 2003b). The models considered in our study have different gravities. This affects the injection length-scale and we assume here that it scales as the pressure scale-height at the photosphere. Such a procedure has been verified in the case of $\alpha$ Cen A (Samadi et al. 2008). This allows us to compute the excitation rates for all the models presented in Sect. 3.1. We use a Lorentzian profile for the eddy time-correlation function (Samadi et al. 2003a). Eventually, concerning the $k$-dependency of the kinetic energy spectrum ( $k$ is the wavenumber in the Fourrier space of turbulence), we use the Broad Kolmogorov Spectrum (BKS) (Musielak et al. 1994) that reproduces the spectrum inferred from the $3 \mathrm{D}$ simulation.

\section{Results}

\subsection{Mode trapping in evolved stars}

Mode trapping is a very important phenomenon allowing to understand the behaviour of non-radial modes in evolved stars. D01 and Christensen-Dalsgaard (2004) discussed it in the case of red giants. Here we recall basic aspects that are important for the interpretation of our results. The Brunt-Väisälä $(N)$ and Lamb $\left(L_{\ell}\right)$ frequencies are huge in the dense core of red giants. Because of these huge values, all non-radial modes with frequencies lower than the cut-off frequency and larger than the fundamental radial mode have a mixed character. In the envelope, $\sigma^{2}>L_{\ell}^{2}, N^{2}$ 


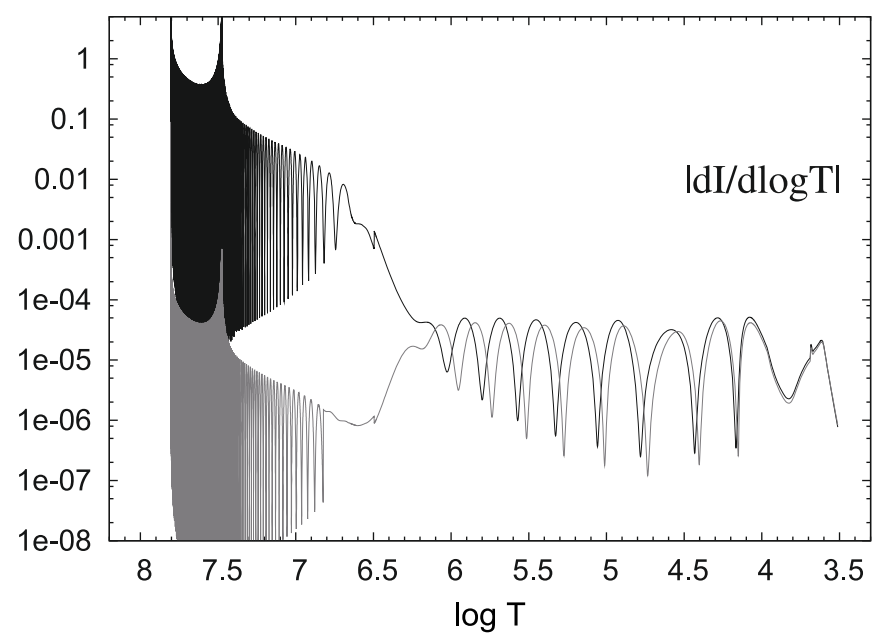

Fig. 2. Energy density $|\mathrm{d} I / \mathrm{d} \log T|$ for the mode $\ell=2, v=55.72 \mu \mathrm{Hz}$ trapped in the envelope (grey) and the mode $\ell=2, v=53.87 \mu \mathrm{Hz}$ trapped in the core (black), for model B.

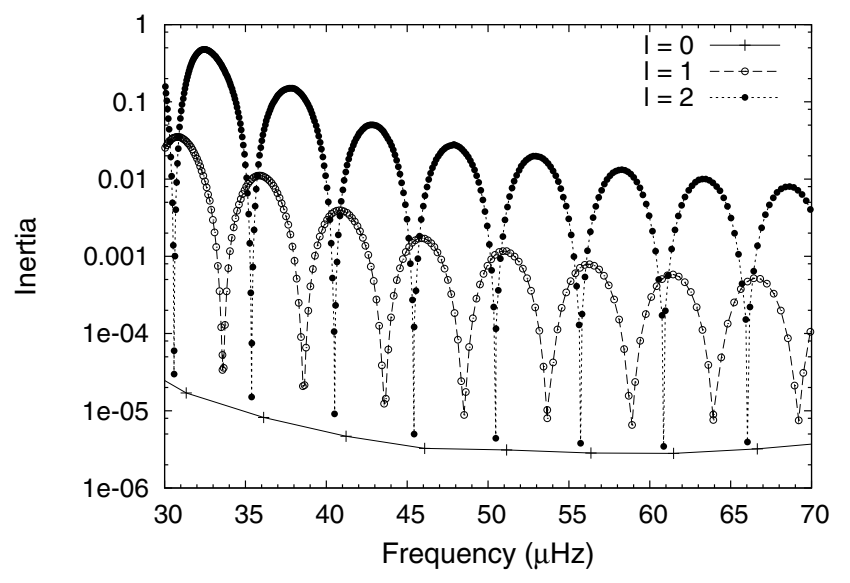

Fig. 3. Inertia of $\ell=0,1$ and 2 modes of model B.

and they behave like acoustic modes (as in solar-like main sequence stars). But $\sigma^{2}<L_{\ell}^{2}, N^{2}$ in the core and the same modes behave there like gravity modes. The presence of an evanescent region between the $\mathrm{p}$ - and g-cavities is at the origin of the mode trapping. Some modes have significant kinetic energy in the gcavity and low ones in the p-cavity; they are trapped in the core. Others have low kinetic energy in the g-cavity but high ones in the envelope; they are trapped in the envelope.

An illustration of the distribution of energy of a mode trapped in the core and a mode trapped in the envelope are shown in Fig. 2, where we give $|\mathrm{d} I / \mathrm{d} \log T|=-|\xi|^{2} \mathrm{~d} m /(M \mathrm{~d} \log T)$. Mode trapping directly affects the mode inertia. We give in Fig. 3 the inertia as a function of frequency for $\ell=0-2$ modes. We first notice how dense the spectrum of frequencies for non-radial modes is, which is a direct consequence of the huge BruntVäisälä frequency in the core (according to the asymptotic theory, $P_{n, \ell}-P_{n-1, \ell} \simeq 2 \pi^{2} /\left(\ell(\ell+1) \int N / r \mathrm{~d} r\right)$. Modes trapped in the envelope are the local minima with low inertia in Fig. 3. The $\ell=2$ modes trapped in the envelope have lower inertia than the $\ell=1$ modes trapped in the envelope, while it is the contrary for all other modes. In agreement with D01 and ChristensenDalsgaard (2004), the size of the evanescent region and thus the efficiency of trapping increases with the degree $\ell$ of the modes.

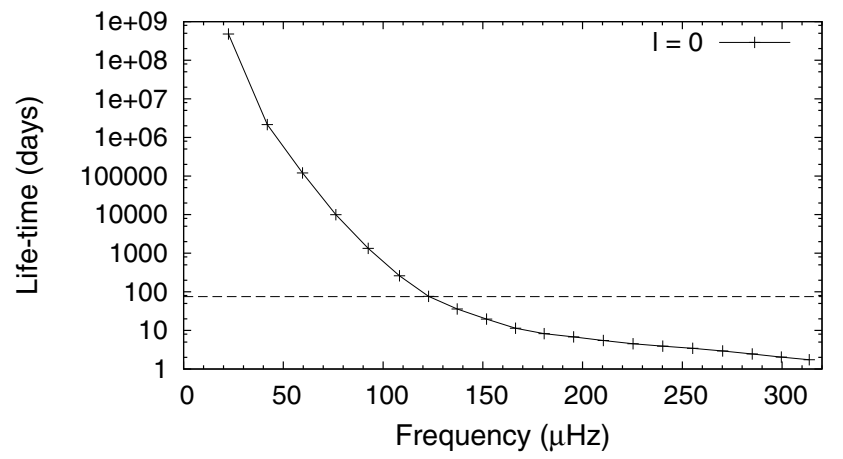

Fig. 4. Theoretical lifetimes of radial modes of model A. The horizontal line corresponds to our choice of the resolution limit ( 75 days).

This is very different from RR Lyrae stars (Van Hoolst et al. 1998) because there the evanescent region is larger for $\ell=1$ in the observed range of frequency. It is very interesting to notice that when only the modes trapped in the envelope are considered, the frequency spectrum is similar to solar-type main sequence stars, with large and small separations appearing clearly. The inertia appears directly in Eqs. (1), (5) and (8). Hence, the mode trapping is expected to strongly affect the amplitudes, lifetimes, and heights of the modes in the PS of red giants. In the next section, we present our main results for different models (Table 1, Fig. 1).

\subsection{Model A: bottom of the red giant branch}

We first consider the lifetimes of radial modes from the fundamental up to the cut-off frequency. We also give the resolution limit of 75 days (horizontal line). As can be seen, we can separate the modes into two sets. From the fundamental to $\mathrm{p}_{7}$ $(v=123 \mu \mathrm{Hz}$ ), the lifetimes are very long and such modes could not be resolved. On the other side, for larger frequencies the lifetimes of radial modes are below 75 days and these radial modes have a chance of being resolved. Current observations of red giant solar-like oscillations show that at least some of the modes can be resolved. We note also that Xiong \& Deng (2007) predict unstable modes in the lower part of the spectrum. Depending on the parameter $\beta$ used in our TDC model, they can be stable or unstable. We restrict our study to the second set of modes, in which radial modes could be resolved. We propose to call them solar-like modes by analogy with the solar case. Such modes are detected (as shown in the introduction) and their physical interpretation as stochastic modes has been firmly established.

In the top panel of Fig. 5, we give the theoretical lifetimes $\tau=1 / \eta$ obtained for the $\ell=0,1$ and 2 modes of model A. According to Eq. (1), the mode lifetime $\tau=1 / \eta \propto I$. Moreover, we see from Eq. (7) that the heights in the PS are proportional to $(\tau / I)^{2}$ for resolved modes. This leads us to consider in the second panel the mode lifetimes divided by the inertia $(\tau / I)$. As we showed in Fig. 3, the mode trapping leads to an oscillatory behaviour of the inertia with the large frequency separation as periodicity. The oscillatory behaviour of the lifetimes is a direct consequence of the oscillations of $I$. But when the ratio between the two is considered, the oscillatory behaviour disappears and the same results are found for radial and non-radial modes.

In order to interpret this result, we give in Fig. 6 the cumulated work integral $W(m)=\int_{0}^{m} \mathrm{~d} W /(2 \sigma I M)$ for a typical $\ell=2$ mode trapped in the core $(v=201.3 \mu \mathrm{Hz})$. The surface value 

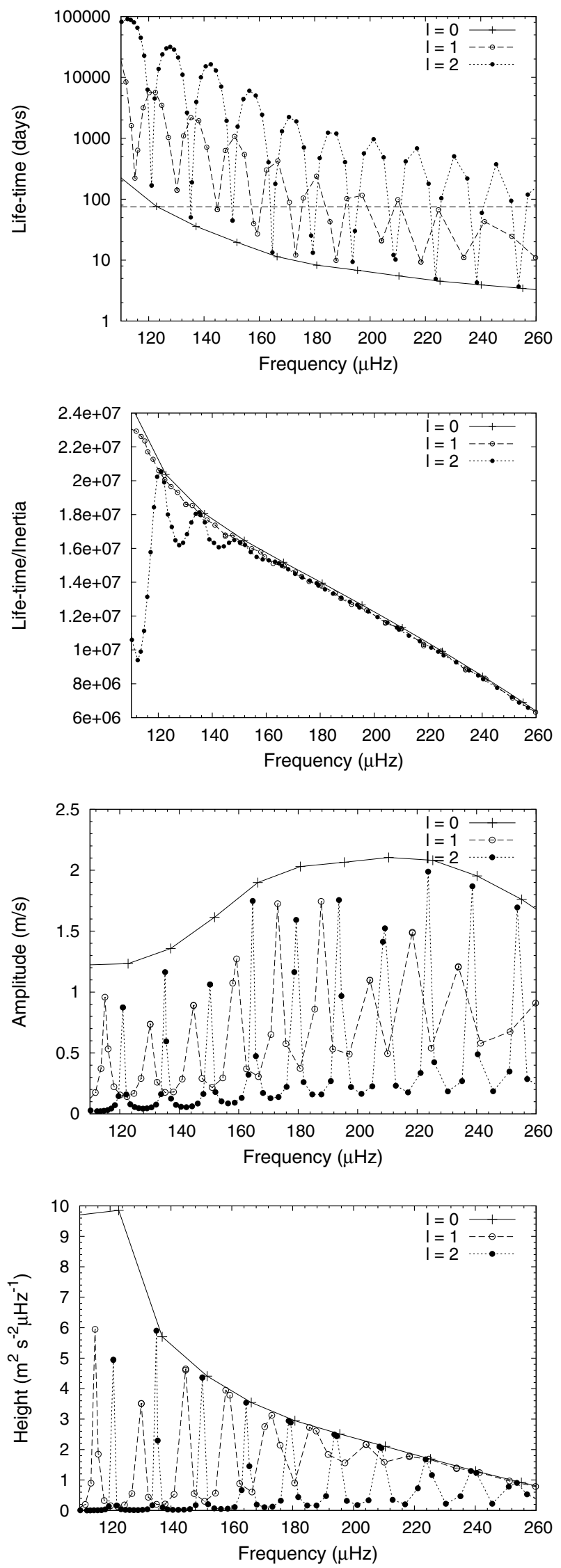

Fig. 5. Theoretical predictions for $\ell=0,1$ and 2 modes of model A. Top panel: theoretical lifetimes; the horizontal line corresponds to our choice of the resolution limit (75 days). Panel 2: lifetimes over inertia $(\tau / I)$. Panel 3: amplitudes $(\mathrm{m} / \mathrm{s})$. Panel 4: heights in the PS.

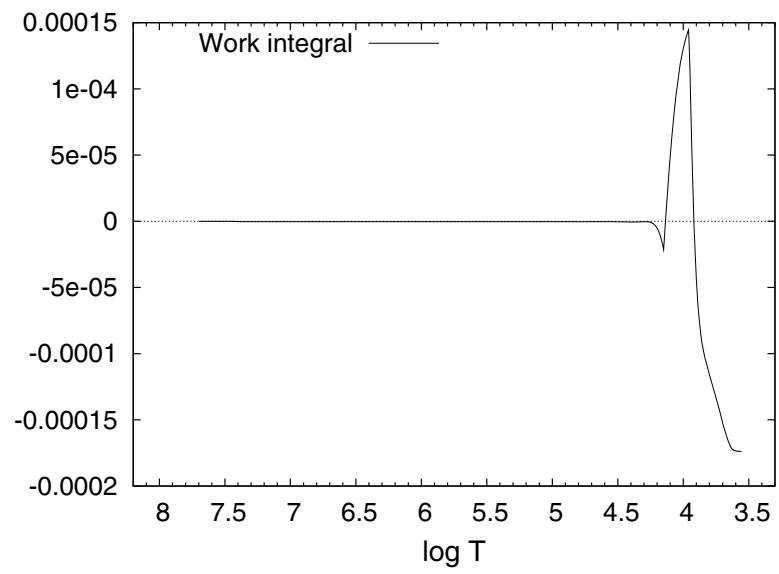

Fig. 6. Work integral for the mode $\ell=2, v=201.3 \mu \mathrm{Hz}$ trapped in the core of model A.

is $-\eta R^{3 / 2} /(G M)^{1 / 2}$. We see that significant driving and damping only occur in the upper part of the convective envelope, but there is no significant radiative damping in the core. As the radial component of the displacement dominates in the envelope, the numerator of Eq. (1) is the same at a given frequency, whatever the value of $\ell$. This explains why $\tau / I$ does not depend on $\ell$.

In the third panel of Fig. 5, we give the theoretical amplitudes of the modes. The amplitudes of radial modes are higher because they have less inertia. The oscillatory behaviour of the inertia due to mode trapping leads to oscillations of the amplitudes of non-radial modes.

In the bottom panel of Fig. 5, we give the theoretical heights in the PS. We put the resolution limit at 75 days (horizontal line in top panel). If the mode lifetime value is larger than this value, Eq. (8) is used instead of Eq. (7) to obtain the height. For resolved modes $\left(H \propto(\tau / I)^{2}\right)$, the same heights are found for radial and non-radial modes, except for the visibility factor (integration over the stellar disk), which is not included in our study. The unresolved non-radial modes with long lifetimes (mainly $\ell=2$ modes trapped in the core) have smaller heights than the closest radial mode. We emphasize that the predictions for the heights are different from the amplitudes. Although non-radial modes have smaller amplitudes than radial modes, the heights in the PS are often of the same order. Contrary to the amplitudes, the heights are observation dependent. If $T_{\text {obs }}$ is smaller, more non-radial modes are unresolved and their heights in the PS are smaller.

Thus, many non-radial modes would be detectable in the PS of stars like model A. Moreover, the interaction between the pand g-mode cavities leads to many avoided crossings. Hence, the resulting frequency spectrum is very complex, particularly for $\ell=1$ modes and its seismic interpretation is not easy.

\subsection{Model B: intermediate in the red giant branch}

We now present the results obtained for model B that is slightly higher in the red giant branch (Fig. 1, Table 1). In the top panel of Fig. 7, we give the theoretical lifetimes obtained for the $\ell=0$, 1 and 2 modes. Again we see the oscillatory behaviour due to mode trapping. But considering now the ratio between the lifetime and the inertia given in the 2nd panel from the top, we find very different results compared to model A. Now, oscillatory behaviour of this ratio is present for non-radial modes, particularly 

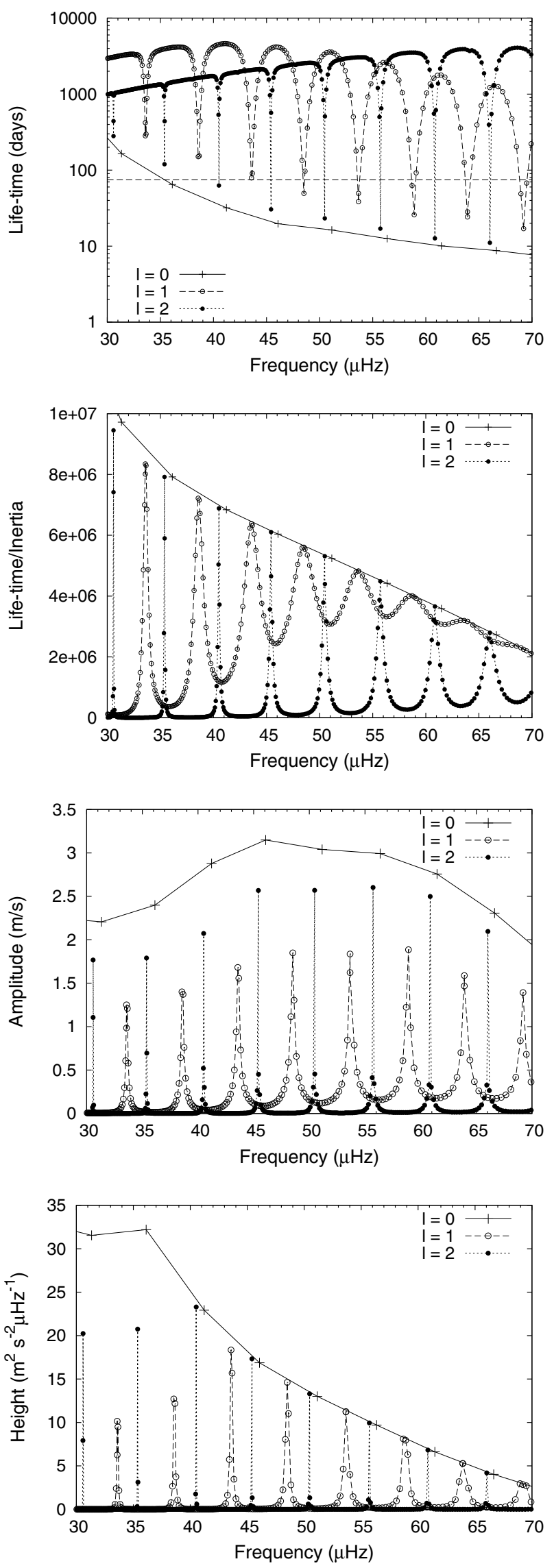

Fig. 7. Theoretical predictions for $\ell=0-2$ modes of model B. Top panel: theoretical lifetimes, panel 2: lifetimes over inertia $(\tau / I)$, panel 3 : amplitudes $(\mathrm{m} / \mathrm{s})$, panel 4 : heights in the PS.
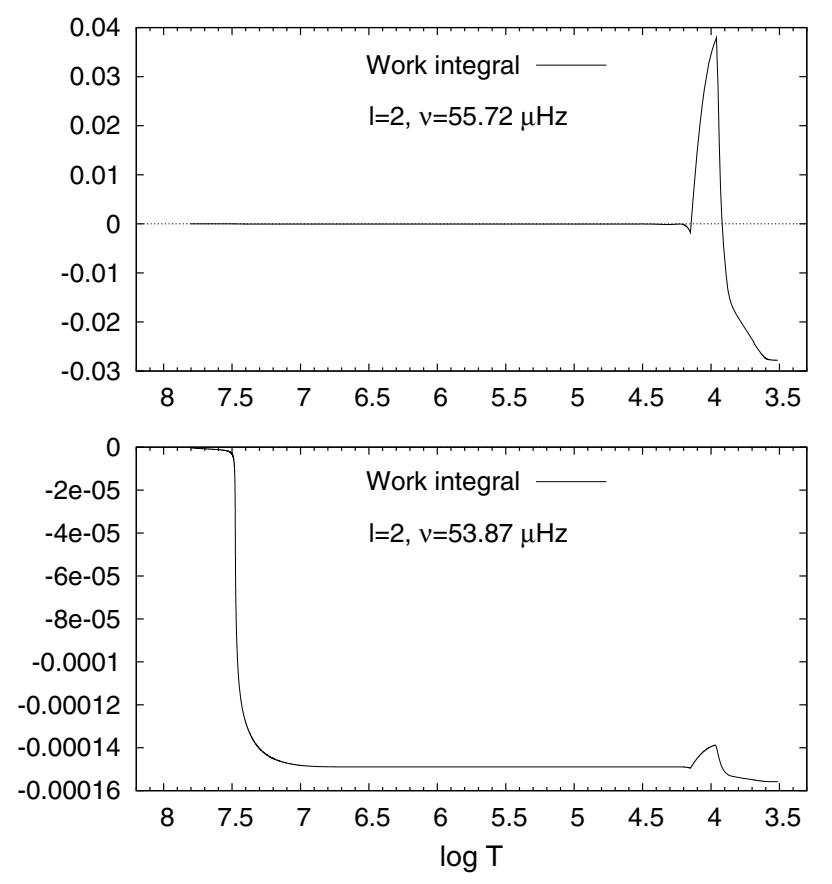

Fig. 8. Work integrals for the mode $\ell=2, v=55.72 \mu \mathrm{Hz}$ trapped in the envelope (top panel) and the mode $\ell=2, v=53.87 \mu \mathrm{Hz}$ trapped in the core (bottom panel) of model B.

at low frequencies. To understand this, we compare in Fig. 8 the work integral for two $\ell=2$ modes, one trapped in the envelope $(v=55.72 \mu \mathrm{Hz}$, top panel) and the other trapped in the core ( $v=53.87 \mu \mathrm{Hz}$, bottom panel). For the mode trapped in the envelope, the driving and damping occur in the upper part of the convective envelope, as in model A. But for the mode trapped in the core, significant radiative damping occurs around the bottom of the H-burning shell $(\log T \simeq 7.5)$.

In the third panel of Fig. 7, we give the theoretical amplitudes of radial and non-radial modes of model B. The amplitudes of non-radial modes trapped in the core are very small because of large inertia and radiative damping. The amplitudes of non-radial modes trapped in the envelope (local maxima in the figure) are smaller than radial modes but not negligible because of similar inertia and negligible radiative damping.

In the bottom panel of Fig. 7, we give the heights of the modes in the PS. Non-radial modes trapped in the core have negligible heights and would not be detected. In contrast, non-radial modes trapped in the envelope have similar heights compared to radial modes.

Thus, only radial modes and non-radial modes trapped in the envelope could be observed in the PS of stars like model B. The groups of non-radial modes trapped in the envelope are more or less separated from each other by a constant large separation. Seismic interpretation of the frequency spectrum of such stars would thus be easier than in model A. We notice however that the trapping is not perfect for $\ell=1$ modes. Hence, a small group of $\ell=1$ modes is detectable around each local maximum. This would have to be taken into account in any seismic study. Considering observed power spectra, this also means that these groups of modes would have to be not confused with single large line-widths modes. 

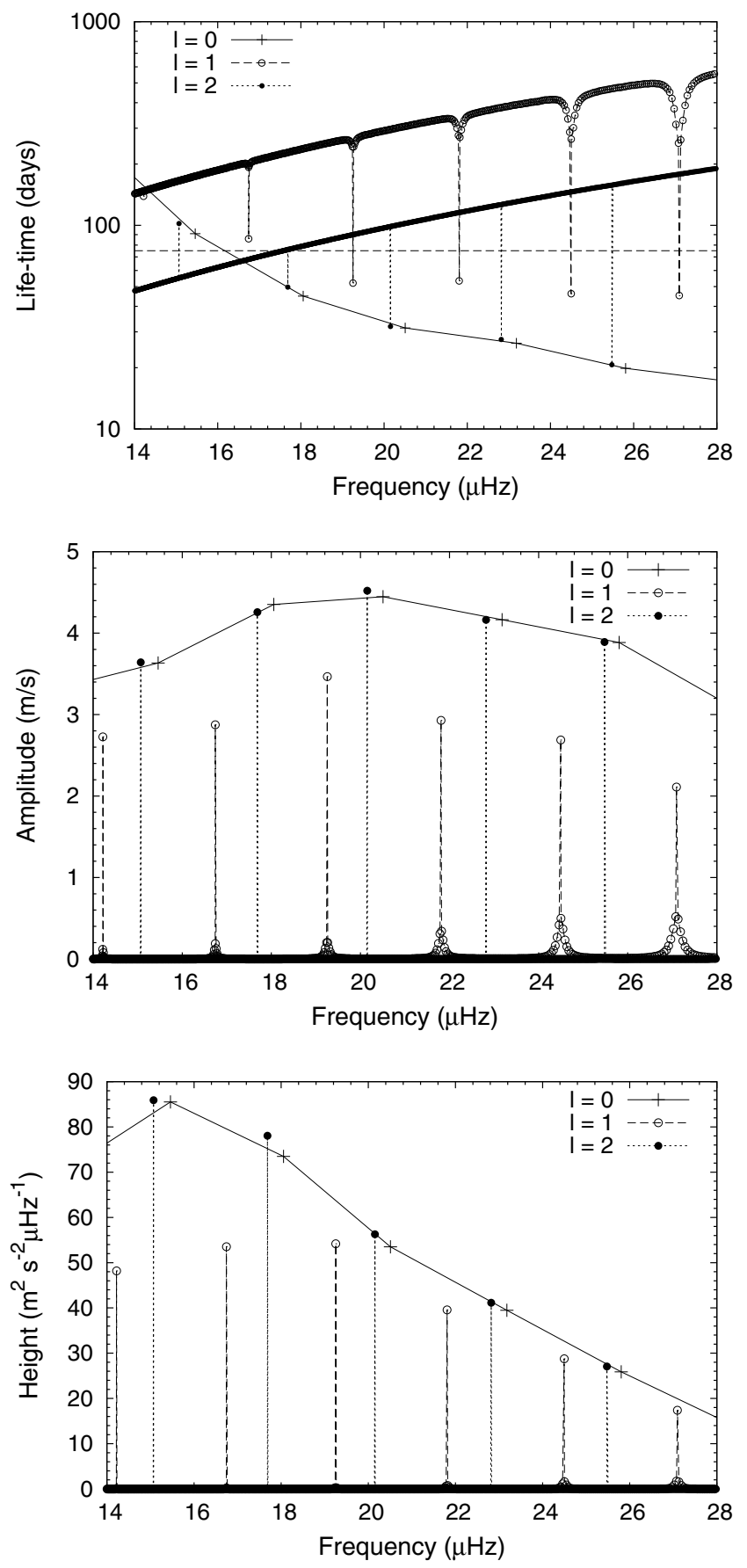

Fig. 9. Theoretical predictions for $\ell=0-2$ modes of model C. Top panel: theoretical lifetimes, panel 2: amplitudes $(\mathrm{m} / \mathrm{s})$, panel 3: heights in the PS.

\subsection{Model C: high in the red giant branch}

We consider now the results obtained for model $\mathrm{C}$, which is even higher in the red giant branch than the previous ones (Fig. 1). In the top panel of Fig. 9, we give the theoretical lifetimes. They are very different from those of model $\mathrm{B}$. The oscillatory behaviour is no longer present for most $\ell=2$ and low frequency $\ell=1$ modes. As can be seen in the figure, the transitions to envelopetrapped modes are very sharp; these modes, known as Strongly Trapped in the Envelope (STE), are nearly perfectly reflected at the internal turning point (near the top of the H-burning shell). We give in Fig. 10 the work integral for an $\ell=2$ mode with

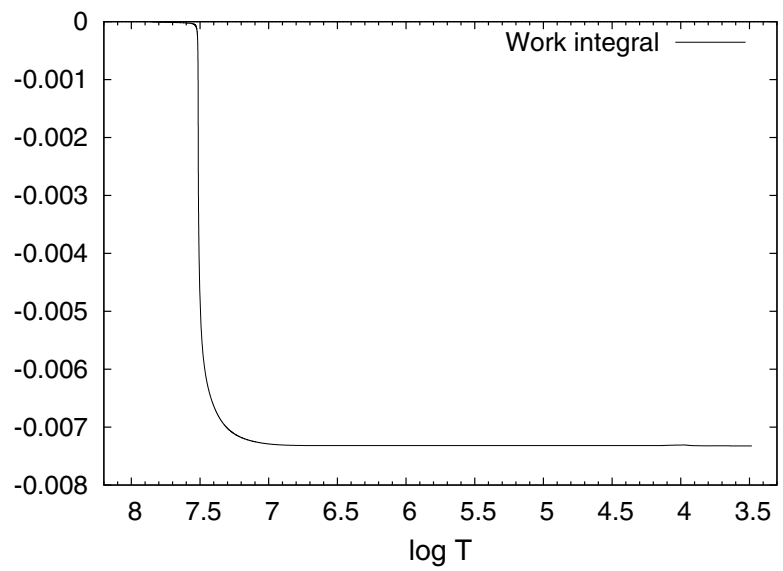

Fig. 10. Work integral for the mode $\ell=2, v=22.83 \mu \mathrm{Hz}$ of model $\mathrm{C}$ (this mode is near a mode trapped in the envelope).

frequency very close to but different from an STE mode. The very high radiative damping illustrated in this figure occurs for all non-radial modes of this model, except the STE mode. Because of this and the large inertia, the g-mode cavity dominates the integrals in the numerator and the denominator of Eq. (1) for all non-STE modes. Hence Eqs. (3) and (4) are good approximations for the full integrals and the $K$ constant simplifies when the ratio between the two is considered. This explains why the lifetimes do not show oscillations in the top panel. In the second panel of Fig. 9, we give the theoretical amplitudes. They are very small for all non-radial non-STE modes, because of the high radiative damping and inertia. Finally, we give in the bottom panel the theoretical heights in the PS. All non-radial non-STE modes have negligible heights because of the large radiative damping. Thus, only radial modes and non-radial modes completely trapped in the envelope could be detected in stars like model C.

\subsection{Models D and E: H-shell versus He-core burning}

We now compare the theoretical lifetimes, amplitudes and heights of the two models D and E with the same luminosity, one before (model D) and the other during (model E) the core helium burning phase.

In Fig. 11, we give the theoretical lifetimes obtained for the $\ell=0-2$ modes of these two models. The behaviour is similar for the two models.

In Fig. 12, we give the theoretical heights in the PS for these two models. No significant difference between the two is found except that the heights in the PS are systematically larger for the He burning model, because of its higher effective temperature leading to stronger stochastic driving.

\subsection{Uncertainties of the non-adiabatic treatment in the convective envelope}

As discussed in Sects. 2.2 and 3.2, the time-dependent interaction between convection and oscillations is subject to large uncertainties. In the TDC treatment of G05, a free complex parameter $\beta$ was introduced in the perturbed closure equations. In the previous computations, we adopted $\beta=-2 i$. We now redo the computations with $\beta=-1.75 i$. Larger changes of $\beta$ would give unstable modes in the considered range of frequencies, which is 

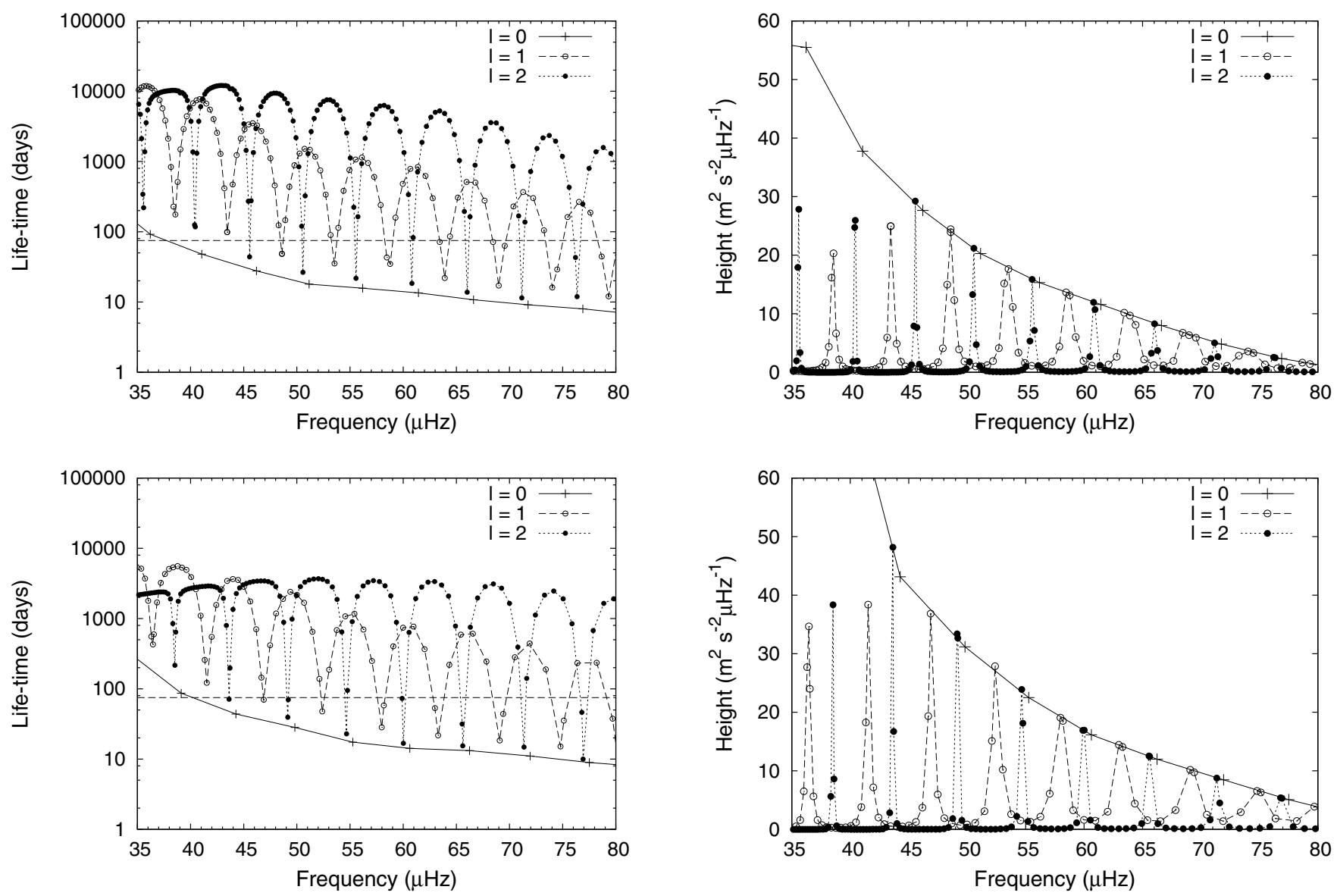

Fig. 11. Theoretical lifetimes for $\ell=0-2$ modes of model D (pre-He burning, top panel) and model E (He burning, bottom panel).

Fig. 12. Theoretical heights in the PS for $\ell=0,2$ modes of model D (top panel) and model E (bottom panel).

\section{Conclusions}

not realistic in view of the typical observed amplitudes of red giant high order $\mathrm{p}$-modes.

With $\beta=-1.75 i$, the convective damping in the upper part of the convective envelope is larger. As shown in Fig. 13, this results in smaller lifetimes and amplitudes of the modes trapped in the envelope compared to Fig. 7. In the upper part of the envelope, the eigenfunctions do not depend significantly on the degree $\ell$ of the modes. Hence the uncertainties associated with the TDC treatment affect radial and non-radial modes trapped in the envelope in the same way.

\subsection{Non-linear effects?}

Our study is linear, raising the question of whether such an approximation is valid here. Considering first the amplitudes of the displacement at the photosphere, we find typical values about 200 times smaller than the pressure scale height for detectable modes. Hence non-linear effects in the superficial layers are expected to be small. We consider now the oscillations in the g-mode cavity. We can confidently neglect non-linear terms if $\left|v_{\mathrm{r}} \partial \boldsymbol{v} / \partial r\right| \ll|\partial \boldsymbol{v} / \partial t|$. In a cavity with short wavelength oscillations, this condition is equivalent to $\left|\xi_{\mathrm{r}}\right| \ll 1 / k_{\mathrm{r}}$. In the $\mathrm{g}$ mode cavity of envelope trapped modes, $\left|\xi_{\mathrm{r}}\right|$ is typically about $10^{4}$ times smaller than $1 / k_{\mathrm{r}}$. Hence the risk of wavebreaking due to non-linear effects is negligible in the core.

We have determined the theoretical lifetimes, amplitudes and heights in the PS of radial and non-radial modes of several red giant models. The predictions appear to be very different depending on the evolutionary status of the star. What mainly matters is the density contrast between the He core and the envelope. During evolution, it increases considerably as the He core contracts and the envelope expands. Moreover the Kelvin-Helmholtz time decreases because of the increasing luminosity. The consequence of these two effects is an increasing importance of the radiative damping of non-radial modes as the luminosity of the star increases. We can encounter very different cases depending on the evolutionary status of the star:

- Case A: Subgiants and/or low luminosity red giants.

Our model representative of the class is model A at the bottom of the giant branch for $2 M_{\odot}$. In these stars, the density contrast is not large enough to imply significant radiative damping of non-radial solar-type p-modes $\left(R_{\mathrm{c}} / R=0.0075\right.$ in model A). The amplitudes of non-radial modes are smaller than radial modes because of larger inertia. However, if we consider instead the heights in the PS, they are similar for radial and non-radial modes as long as they can be resolved. As the heights are used as detection criteria in a Fourier analysis, many non-radial modes could be detected in the PS of such stars. The interaction between the p- and gmode cavities leads to many avoided crossings. The resulting 

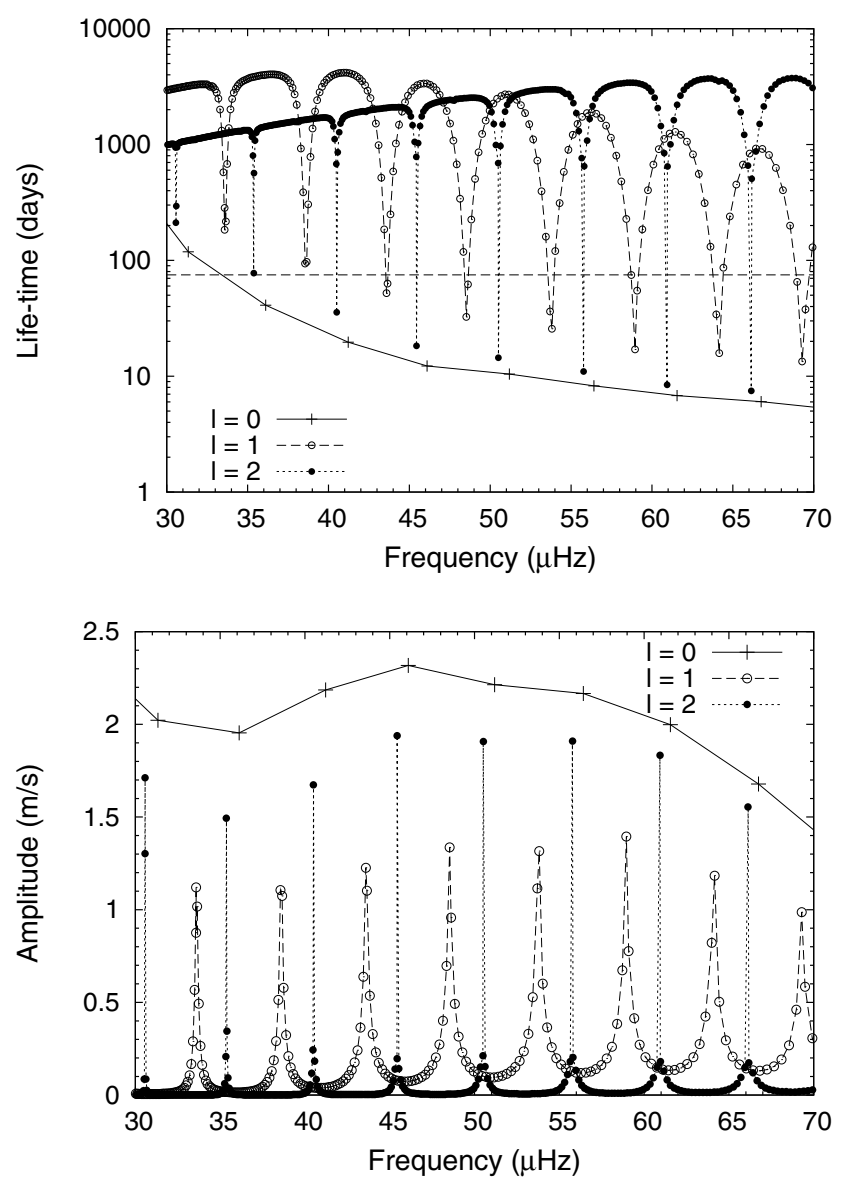

Fig. 13. Theoretical predictions for $\ell=0,1$ and 2 modes of model $\mathrm{B}$, but with another value of the free parameter of our TDC treatment $\beta=$ -1.75 i. Top panel: theoretical lifetimes, bottom panel: heights in the PS.This figure can be compared with Fig. 7 for which $\beta=-2 i$.

frequency spectrum is thus very complex. Asteroseismology of such stars would be challenging.

- Case B: Intermediate models in the red giant branch.

Our models representative of this class are model B, D and E with greater luminosity compared to the previous case $\left(\log \left(L / L_{\odot}\right)=1.8-2\right)$. In these stars the radius ratio between the core and the envelope is smaller $\left(R_{\mathrm{c}} / R=0.003\right.$ in model B). As a consequence, the radiative damping around the bottom of the H-burning shell becomes large enough to destroy the non-radial modes trapped in the core. Only the non-radial modes trapped in the envelope and the radial modes can survive and have similar heights in the PS. The non-radial modes trapped in the envelope are more or less separated from each other by a constant large separation, allowing us to build echelle diagrams. However, the trapping is not perfect for $\ell=1$ modes. Hence, a small group of $\ell=1$ modes is detectable around each local maximum in the PS corresponding to an envelope trapped mode. Such structures would not have to be interpreted as single large linewidth modes. Seismic interpretation of the frequency spectrum of such stars would be easier than in the previous case.

- Case C: High luminosity red giants.

Our model representative of this class is model $\mathrm{C}$ with $M=2 M_{\odot}, \log \left(L / L_{\odot}\right)=2.1$. This case is not very different from the previous one, except that the density contrast between the core and the envelope is very large $\left(R_{\mathrm{c}} / R=0.002\right.$ in model $\mathrm{C}$ ). As a consequence, the radiative damping is stronger in the core and destroys all non-radial modes except those strongly trapped in the envelope. These modes and the radial modes are the only ones that could be detected in these stars. The trapping is efficient enough in $\ell=1$ modes and only single envelope trapped modes are detectable. Seismic interpretation of the frequency spectrum of such stars would also be easier.

We also notice that the duration of observation is very important, as it determines the frontier between resolved and unresolved modes. Non-radial modes have always much longer lifetimes $(\tau)$ than radial modes because of their greater inertia. If $\tau \gtrsim T_{\text {obs }} / 2$, the modes cannot be resolved and have much smaller heights in the PS. With too short observations, as is typically the case with ground based observations, only radial and strongly trapped non-radial modes have a chance of being detected. On the other hand, future space missions will provide longer observations: e.g. 5 years for Kepler, 3 years for Plato, increasing the ability to detect more non-radial modes.

Asteroseismology of red giants is a very promising field as the theoretical predictions depend strongly on the physical characteristics of both their deep and superficial layers.

Acknowledgements. The works of K. Belkacem were financially supported throught a postdoctoral fellowship from the "Subside fédéral pour la recherche 2009”, University of Liège. We thank Prof. W. Dziembowski for his useful advices and remarks.

\section{References}

Alexander, D. R., \& Ferguson, J. W. 1994, ApJ, 437, 879

Angulo, C., Arnould, M., Rayet, M., et al. 1999, Nuclear Physics A, 656, 3

Balmforth, N. J. 1992, MNRAS, 255, 603

Barban, C., Matthews, J. M., de Ridder, J., et al. 2007, A\&A, 468, 1033

Baudin, F., Samadi, R., Goupil, M.-J., et al. 2005, A\&A, 433, 349

Bedding, T., \& Kjeldsen, H. 2006, in Proceedings of SOHO 18/GONG 2006/HELAS I, Beyond the spherical Sun, ESA SP, 624

Belkacem, K., Samadi, R., Goupil, M. J., Kupka, F., \& Baudin, F. 2006, A\&A, 460,183

Belkacem, K., Samadi, R., Goupil, M.-J., \& Dupret, M.-A. 2008, A\&A, 478, 163 Berthomieu, G., Toutain, T., Gonczi, G., et al. 2001, in SOHO 10/GONG 2000 Workshop: Helio- and Asteroseismology at the Dawn of the Millennium, ed. A. Wilson, \& P. L. Pallé, ESA SP-464, 411

Böhm-Vitense, E. 1958, Z.Astrophys., 46, 108

Chaplin, W. J., Houdek, G., Elsworth, Y., et al. 2005, MNRAS, 360, 859

Christensen-Dalsgaard, J. 2004, Sol. Phys., 220, 137

De Ridder, J., Barban, C., Carrier, F., et al. 2006, A\&A, 448, 689

De Ridder, J., Barban, C., Baudin, F., et al. 2009, Nature, 459, 398

Dupret, M.-A. 2002, Bull. Soc. Roy. Sc. Liège, 5, 249

Dupret, M.-A., Grigahcène, A., Garrido, R., Gabriel, M., \& Scuflaire, R. 2004, A\&A, 414, L17

Dupret, M. A., Barban, C., Goupil, M.-J., et al. 2006a, in Proceedings of SOHO 18/GONG 2006/HELAS I, Beyond the spherical Sun, ESA SP, 624

Dupret, M.-A., Samadi, R., Grigahcene, A., Goupil, M.-J., \& Gabriel, M. 2006b, Commun. Asteroseismol., 147, 85

Dziembowski, W. 1977a, Acta Astron., 27, 203

Dziembowski, W. 1977b, Acta Astron., 27, 95

Dziembowski, W. A., Gough, D. O., Houdek, G., \& Sienkiewicz, R. 2001, MNRAS, 328, 601

Frandsen, S., Carrier, F., Aerts, C., et al. 2002, A\&A, 394, L5

Freytag, B., Steffen, M., \& Dorch, B. 2002, Astron. Nachr., 323, 213

Gabriel, M. 1996, BAS of India, 24, 233

Godart, M., Noels, A., Dupret, M.-A., \& Lebreton, Y. 2009, MNRAS, 396, 1833 Gough, D. O. 1977a, in Problems of Stellar Convection, ed. E. A. Spiegel \& J.-P. Zahn (Berlin: Springer Verlag), Lect. Notes Phys., 71, 15

Gough, D. O. 1977b, ApJ, 214, 196

Grevesse, N., \& Noels, A. 1993, in La formation des éléments chimiques, AVCP, ed. R. D. B. Hauck, \& S. Paltani, 205

Grigahcène, A., Dupret, M.-A., Gabriel, M., Garrido, R., \& Scuflaire, R. 2005, A\&A, 434, 1055

Hekker, S., Aerts, C., de Ridder, J., \& Carrier, F. 2006, A\&A, 458, 931 
Henyey, L., Vardya, M. S., \& Bodenheimer, P. 1965, ApJ, 142, 841

Houdek, G. 2000, in Delta Scuti and Related Stars, ed. M. Breger, \& M. Montgomery, ASP Conf. Ser., 210, 454

Houdek, G., \& Gough, D. O. 2002, MNRAS, 336, L65

Iglesias, C. A., \& Rogers, F. J. 1996, ApJ, 464, 943

Kallinger, T., Guenther, D. B., Matthews, J. M., et al. 2008a, A\&A, 478, 497

Kallinger, T., Guenther, D. B., Weiss, W. W., et al. 2008b, Commun. Asteroseismol., 153, 84

Lochard, J., Samadi, R., \& Goupil, M. J. 2005, A\&A, 438, 939

Mazzitelli, I. 1979, A\&A, 79, 251

Musielak, Z. E., Rosner, R., Stein, R. F., \& Ulmschneider, P. 1994, ApJ, 423, 474

Potekhin, A. Y., Baiko, D. A., Haensel, P., \& Yakovlev, D. G. 1999, A\&A, 346, 345

Rogers, F. J., \& Nayfonov, A. 2002, ApJ, 576, 1064

Samadi, R., \& Goupil, M. 2001, A\&A, 370, 136

Samadi, R., Nordlund, Å., Stein, R. F., Goupil, M. J., \& Roxburgh, I. 2003a, A\&A, 404, 1129
Samadi, R., Nordlund, Å., Stein, R. F., Goupil, M. J., \& Roxburgh, I. 2003b, A\&A, 403, 303

Samadi, R., Belkacem, K., Goupil, M. J., Dupret, M.-A., \& Kupka, F. 2008, A\&A, 489, 291

Saumon, D., Chabrier, G., \& van Horn, H. M. 1995, ApJS, 99, 713

Spiegel, E. A. 1963, ApJ, 138, 216

Stello, D., Kjeldsen, H., Bedding, T. R., \& Buzasi, D. 2006, A\&A, 448, 709

Stolzmann, W., \& Bloecker, T. 1996, A\&A, 314, 1024

Unno, W. 1967, PASJ, 19, 140

Van Hoolst, T., Dziembowski, W. A., \& Kawaler, S. D. 1998, MNRAS, 297, 536

Ventura, P., Zeppieri, A., Mazzitelli, I., \& D’Antona, F. 1998, A\&A, 334, 953

Ventura, P., D'Antona, F., \& Mazzitelli, I. 2008, Ap\&SS, 316, 93

Wedemeyer, S., Freytag, B., Steffen, M., Ludwig, H.-G., \& Holweger, H. 2004, A\&A, 414, 1121

Xiong, D. R., \& Deng, L. 2001, MNRAS, 324, 243

Xiong, D. R., \& Deng, L. 2007, MNRAS, 378, 1270

Xiong, D. R., Cheng, Q. L., \& Deng, L. 1997, ApJS, 108, 529

Xiong, D. R., Cheng, Q. L., \& Deng, L. 2000, MNRAS, 319, 1079 